\title{
Gamma Non-Ionizing Energy Loss: Comparison with the Damage Factor in Silicon Devices
}

\author{
E. El allam ${ }^{1}$, C. Inguimbert ${ }^{2}$, A. Meulenberg ${ }^{3}$, A. Jorio ${ }^{1}$, I. Zorkani ${ }^{1}$ \\ ${ }^{1}$ Solid State Physics Laboratory, Faculty of Sciences Dhar el Mahraz, University Sidi \\ Mohamed Ben Abdellah, P.B 1796 Atlas-Fez, Morocco \\ ${ }^{2}$ ONERA-DPhIEE, 2 avenue E. Belin, 31055 Toulouse, France \\ ${ }^{3}$ Science for Humanity Trust, Inc. Tucker, GA, USA
}

\begin{abstract}
The concept of non-ionizing energy loss (NIEL) has been demonstrated to be a successful approach to describe the displacement damage effects in silicon materials and devices. However, some discrepancies exist in the literature between experimental damage factors and theoretical NIELs. ${ }^{60} \mathrm{Co}$ gamma rays having a low NIEL is an interesting particle source that can be used to validate the NIEL scaling approach. This paper presents different ${ }^{60} \mathrm{Co}$ gamma ray NIEL values for silicon targets. They are compared with radiation-induced increase in thermal generation rate of minority carriers per unit fluence. The differences between the different models, including one using molecular dynamics, are discussed.
\end{abstract}

\section{INTRODUCTION}

In matter, incident energetic particles (i.e., charged and uncharged particles) lose their energy through a variety of interactions, which result in two main processes. Particles can lose their energy by ionization that is the consequence of the collision between the energetic particle and atomic electrons leading to excitation or ionization (i.e., emission of atomic electrons). The second process is the consequence of the interactions with nuclei that result in atomic displacement or displacement damage (i.e., displace a target atom from its normal lattice position). This type of interaction is quantified by the NIEL (Non Ionizing Energy Loss). Generally, the part of the total energy loss of an incident particle by non-ionizing processes is very small compared to the energy loss by ionization. However in certain cases, the displacement damage could be the main contributor to the degradation of some electrical properties in electronic devices. The radiation, by means of atomic displacement, introduces new stable defect levels in the bandgap of semiconductor that affect the transport of carrier in the semiconductor materials [1]. In many cases, the degradation related to these defects is demonstrated to be proportional to the NIEL.

The parameters of bulk material and devices (e.g., diodes and solar cells) that are most sensitive to displacement damage effects are the minority carrier lifetime, diffusion length, mobility, and carrier concentration. The changes in these parameters, most of time proportional to the particle fluence, allows one to extract a damage factor $\left(\mathrm{K}_{\mathrm{d}}\right)$ that depends mainly of the type and energy of the incident 
particle. The measured degradation could also be affected by the material type ( $\mathrm{n}$ or $\mathrm{p}$ ), impurity type, concentration, fabrication technique, irradiation temperature, temperature and time at which the measurements are made (i.e., effect of annealing, which leads to recovery of defects generated by radiation) [2], and the effects of non-uniform radiation damage [3]. This increases the difficulty of using theoretical calculations to predict accurately the radiation effects on different materials and devices.

Numerous investigations have been made on the correlation of displacement damage produced by various particle types and energies in silicon [1, 4-9]. The results of these studies demonstrate that experimental damage factors can be estimated reasonably well by using the theoretical calculations of the NIEL, which describes the fraction of the total incident particle energy that is imparted to a material to produce displacement damage $[6,10,11]$. However, in some cases the NIEL will fail to reproduce the experimental data $[1,6,12]$. Some discrepancies between NIEL and measured damage factors have been observed in different materials. In the case of silicon material, a linear correlation between the measured $\mathrm{K}_{\mathrm{d}}$ and NIEL is found for heavy particles (heavy ions, protons) and neutrons [1, 5-9]. But, in the case of electrons and gamma rays, a significant deviation from linearity of $K_{d}$ with the NIEL calculations is observed [1, 6-9]. Because of the greater availability of electron accelerators and gamma sources for laboratory testing and simulation of radiation environments and effects, some efforts focused on developing the NIEL prediction for both electrons and gamma rays [13-18] have been performed. Inguimbert et al. [13] proposed a new approach that includes molecular dynamic results, which could explain the quadratic dependence of the damage factor found in silicon following electron irradiation [9]. The NRL group [14-16], addresses this issue by introducing a power factor $n$ (i.e., the fitting parameter) to the NIEL. The value of $n$ is experimentally determined, and varies from one device to another and depends on the type of measured electrical parameter, its values range between 0.5 and 3 [16].

The primary goal of this paper is to present the gamma ray NIEL calculations for silicon. These calculations can be used for predicting the displacement damage effects, due to $\mathrm{C}^{60}$ gamma ray irradiation in silicon devices, without the use of a power factor $n$. There are certain types of radiationinduced displacement damage to semiconductors that cannot be reproduced by either gamma radiation or non-relativistic electrons. These include loose and tight cluster defects, from end-point energies of protons and heavy ions respectively[12], and linear paths of displacement defects, from intense ionization produced by highly-ionized cosmic rays or energetic knock-on lattice ions $[19,20]$. They are generally not a significant source of total device degradation in most radiation environments; however, they do occur and, if significant, they must be addressed separately. Nevertheless, the modification to the NIEL calculation suggested here may be a practical step in resolving some issues with the model. 


\section{THE CALCULATION METHOD OF ${ }^{60} \mathrm{CO}$ GAMMA RAYS NIEL.}

The passage of gamma rays through matter and devices causes ionization and the production of energetic secondary electrons via the photoelectric effect, Compton scattering, and pair production processes. The observed degradation in some materials and devices following ${ }^{60} \mathrm{Co}$ gamma ray irradiation is due primarily to displacement damage from the secondary electrons generated by gamma rays. For instance, gamma rays produced from a ${ }^{60} \mathrm{Co}$ source having an average energy of $1.25 \mathrm{MeV}$, can generate a secondary electrons spectrum ranging up to $1.2 \mathrm{MeV}$ in the shielding material that surrounds the test device. These secondary electrons are slowed down by shielding before they leave to produce displacement damage in the active region of the device. In order to calculate the NIEL of ${ }^{60} \mathrm{Co}$ gamma rays $\left({ }^{60} \mathrm{Co}\right.$ NIEL $\gamma$ ) we have to integrate the slowed down spectrum of secondary electrons with the amount of atomic displacements produced by these secondary electrons (i.e., the electrons NIEL), the gamma NIEL is expressed as [18].

$$
N I E L \gamma=\int_{0}^{E_{\max }} \frac{d S_{c}}{d E}(E) \quad N I E L_{e}(E) d E
$$

Where $d S_{c} / d E$ is the slowed down spectrum of secondary electrons (Fig.1) and $N I E L_{e}(E)$ is the electrons NIEL (Fig.2) at a given secondary energy E.

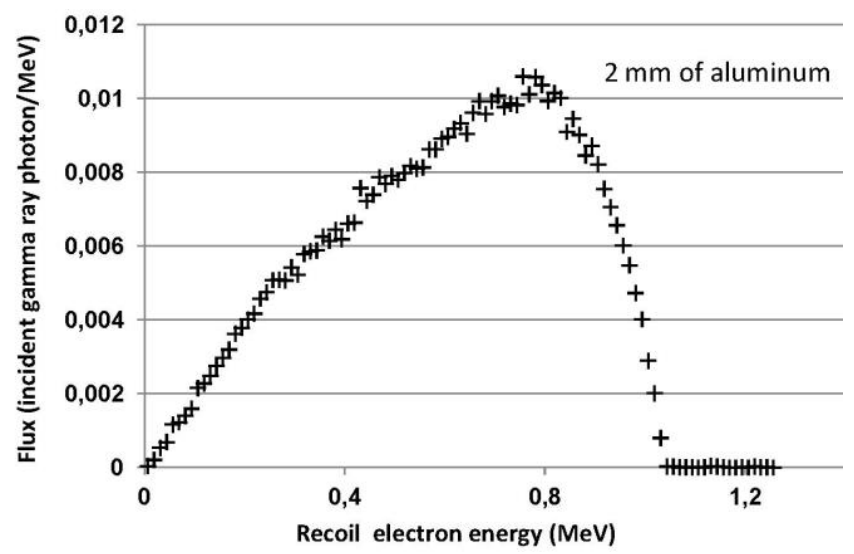

Fig.1 Slowed down spectrum of secondary electrons generated by gamma rays $(1.25 \mathrm{MeV})$ in the aluminum shielding with a thickness of $2 \mathrm{~mm}$. The spectrum is normalized per incident photon.

Monte Carlo simulations are broadly used to simulate the passage of incident particles through matter, because of its flexibility and accuracy. In this work, the Monte Carlo transport code GEANT4 [21] is applied to compute the slowed down spectra of secondary electrons generated by $\mathrm{Co}^{60}$ gamma rays in aluminum. This code provides a good description of the interactions that can occur between the primary gamma source and target, using the Electromagnetic Standard Physics model. Usually, in gamma ray irradiation experiments, the samples must be shielded during irradiation by proper material to achieve a secondary charged-particle equilibrium. It is recommended that shielding thickness be equal to the practical range of the maximum energy of secondary electrons, which can be calculated from the method described in [22]. For example, the maximum secondary electrons energy produced by $\mathrm{Co}^{60}$ gamma rays is approximately $1.2 \mathrm{MeV}$. To ensure equilibrium, the samples can be surrounded by $2 \mathrm{~mm}$ of aluminum shielding. Therefore, the principle of the calculation is to send a unidirectional 
beam of monoenergetic gamma rays $(1.25 \mathrm{MeV})$ into aluminum shielding with a thickness of $2 \mathrm{~mm}$, then the energy spectrum of the secondary electrons exiting the shielding is determined (i.e., the slowed down spectrum that irradiates the active region) as shown in Fig. 1. This slowed down spectrum can vary, if a device or tested material is surrounded by heavy materials, and also depends on the thickness of such materials. It has been reported that the difference in equilibrium spectra between lighter materials and heavy materials could be up to $40 \%$ [18].

TABLE I

NIEL VALUES FOR $\mathrm{Co}^{60}$ GAMMA RAYS IN SILICON

\begin{tabular}{|l|c|c|}
\hline \multicolumn{1}{|c|}{ References } & $\begin{array}{c}\text { The threshold } \\
\text { displacement energies }\end{array}$ & $\begin{array}{c}\mathrm{Co}^{60} \\
\text { gamma rays NIEL } \\
\left(\mathrm{MeV} . \mathrm{cm}^{2} / \mathrm{g}\right)\end{array}$ \\
\hline This study & $\mathrm{T}_{\mathrm{d}, \mathrm{MD}}=1.617 \mathrm{eV}$ & $5,00 \mathrm{E}-09$ \\
\hline This study & $\mathrm{T}_{\mathrm{d}}=12.9 \mathrm{eV}$ & $1,79 \mathrm{E}-07$ \\
\hline This study & $\mathrm{T}_{\mathrm{d}}=21 \mathrm{eV}$ & $1,11 \mathrm{E}-07$ \\
\hline Summers $[6]$ & $\mathrm{T}_{\mathrm{d}}=21 \mathrm{eV}$ & $1,30 \mathrm{E}-05$ \\
\hline Akkerman $[10]$ & $\mathrm{T}_{\mathrm{d}}=21 \mathrm{eV}$ & $1,07 \mathrm{E}-07$ \\
\hline Shatalov $[25]$ & $\mathrm{T}_{\mathrm{d}}=21 \mathrm{eV}$ & $1,83 \mathrm{E}-07$ \\
\hline
\end{tabular}

Fig. 2 shows different NIEL for electrons in silicon that will be used to estimate the ${ }^{60} \mathrm{Co}$ NIEL $\gamma$. Some classical NIEL values, based on the Binary Cascade Approximation (BCA), are shown using two different threshold displacement energies $\left(\mathrm{T}_{\mathrm{d}}=12.9 \mathrm{eV}\right.$ and $\left.\mathrm{T}_{\mathrm{d}}=21 \mathrm{eV}\right)$. These calculations have been performed using the NEMO code [11]. There are other electron NIEL calculations for silicon using the BCA approach $[6,10,23,24]$, and they are in agreement with those calculated by the NEMO code. The "effective" NIEL based on some molecular dynamic simulation results is also plotted in this figure [13]. The effective NIEL is demonstrated to follow, as a function of energy, the classical NIEL to the 2.5 power (Fig. 2). This effective NIEL has been shown to be well correlated with some damage factors at low electron energies [9].

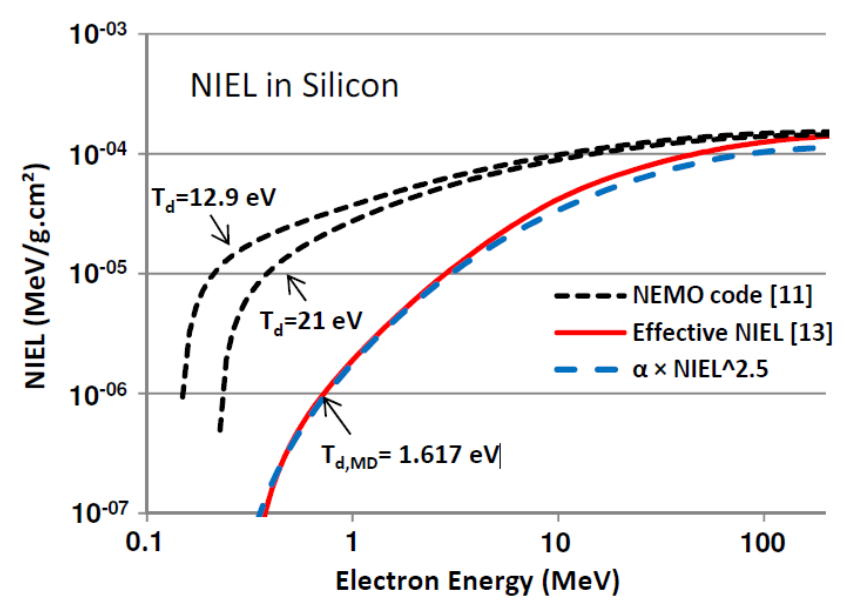

Fig.2. Different electron NIEL curves from classical NIEL [11] and effective NIEL calculations [13] that will be used to calculate the ${ }^{60}$ Co NIEL $\gamma$. The long-dashed curve corresponding to the classical NIEL to the 2.5 power is also plotted, where $\alpha$ is proportionality constant. 
According to Eq. (1), the results represented in Figs. 1 and 2 are used to calculate the ${ }^{60}$ Co NIEL $\gamma$ in silicon, with three different values of threshold displacement energies $\left[\mathrm{T}_{\mathrm{d}}=12.9 \mathrm{eV}, \mathrm{T}_{\mathrm{d}}=21 \mathrm{eV}\right.$, and $\left.\mathrm{T}_{\mathrm{d}, \mathrm{MD}}=1.617 \mathrm{eV}\right]$. Where $\mathrm{T}_{\mathrm{d}}$ is the minimum energy of the primary knock-on atom (PKA) required to displace the target atom from its lattice position; the conventionally used $T_{d}$ value for silicon is $21 \mathrm{eV}$ [26], but the $T_{d}=12.9 \mathrm{eV}$ is sometimes cited as an appropriate value for silicon [27]. The definition of $\mathrm{T}_{\mathrm{d}, \mathrm{MD}}$ is different from $\mathrm{T}_{\mathrm{d}}$. Some molecular dynamic simulations have shown that a defect can be produced even if the energy transferred to the PKA is lower than the threshold displacement energy $T_{d}$ [13]. A defect can result from the recrystallization of a disordered region before it can return to its normal state. $\mathrm{T}_{\mathrm{d}, \mathrm{MD}}$ represents the minimum energy that must be imparted to all the atoms of this damaged region. Our calculations and values given by other authors for the ${ }^{60} \mathrm{Co}$ NIEL $\gamma$ in silicon are listed in Table I. When the same threshold displacement energy $\left(\mathrm{T}_{\mathrm{d}}=21 \mathrm{eV}\right)$ is used, our NIEL value is very close to the value calculated by Akkerman et al [10] ( 4\% difference), and it's a little different from the value presented by Shatalov et al [25] ( 35\% difference). It differs by a factor of about 100 with the ${ }^{60} \mathrm{Co}$ NIEL $\gamma$ value given by Summers et al [6] that represents the NIEL for the average electron energy in the slowed down spectrum of secondary electrons produced by incident ${ }^{60} \mathrm{Co}$ gamma rays. By looking at the Table 1, one observes that the gamma ray NIEL is strongly dependent on the $\mathrm{T}_{\mathrm{d}}$ value; it increases when the $\mathrm{T}_{\mathrm{d}}$ value goes from $21 \mathrm{eV}$ to $12.9 \mathrm{eV}$. In addition, our ${ }^{60} \mathrm{Co}$ NIEL $\gamma$ values calculated using the classical NIEL curves $\left(T_{d}=12.9 \mathrm{eV}\right.$ and $\left.T_{d}=21 \mathrm{eV}\right)$ are found to be a factor of 22 and 36 greater than that calculated using the effective NIEL curve $\left(\mathrm{T}_{\mathrm{d}, \mathrm{MD}}=1.617 \mathrm{eV}\right)$, respectively. Based on the foregoing, to use the NIEL in estimating the effect of gamma rays on materials and devices, it is necessary to takes into account three parameters; the nature and the thickness of shielding surrounding the test material as well as the threshold displacement energy.

\section{COMPARISON OF NIEL WITH DAMAGE FACTOR}

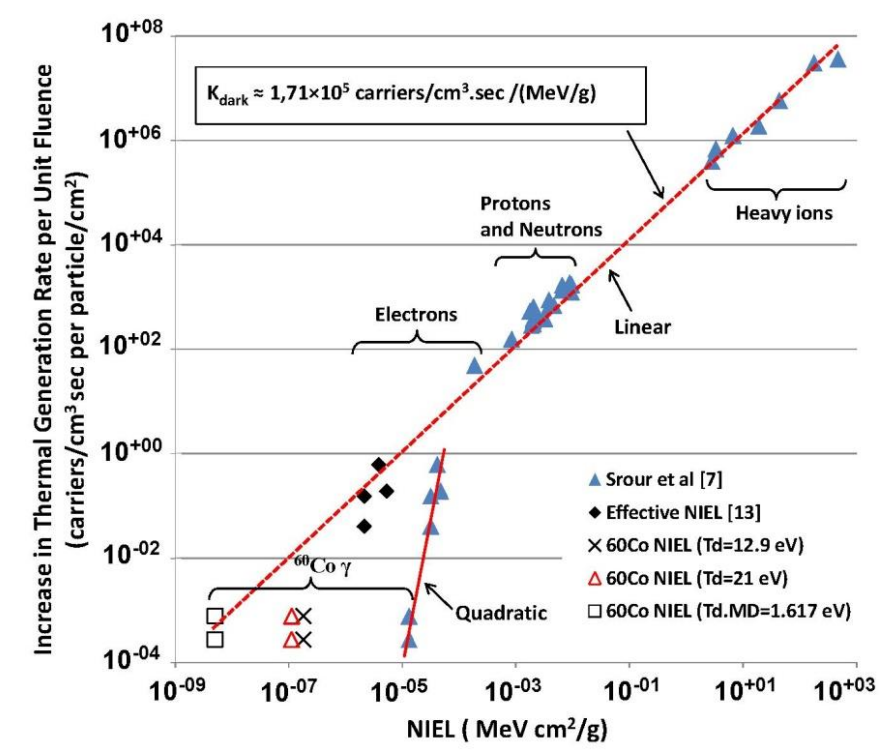

Fig.3. Radiation-induced increase in thermal generation rate per unit fluence $\left(=K_{\text {dark }} \times\right.$ NIEL) as a function of corresponding NIEL values. 
The NIEL presented in the previous section has been compared to some experimental measurements. Srour et al [7] presents a large set of experimentally measured damage factors for various particle types and energies. They found for protons, neutrons and heavy ions a linear behavior of the damage factor as a function of the NIEL. They deduced from this analysis the value of an universal dark-current damage factor $\left(\mathrm{K}_{\text {dark }}\right)$ valid for a variety of silicon devices. It represents the increase in thermal generation rate $(\Delta G)$ rate of carriers per unit deposited displacement damage dose $(\mathrm{DDD}=\mathrm{NIEL} \times$ fluence $)$ [7]. This damage factor is independent of the type of incident particles, except for electrons and gamma rays and it is dependent on the irradiation temperature and time after irradiation. The $\mathrm{K}_{\text {dark }}$ value proposed was $1.9 \times 10^{5}$ carriers $/ \mathrm{cm}^{3} . \mathrm{sec} /(\mathrm{MeV} / \mathrm{g})$ at $300 \mathrm{~K}$ and 1 week after irradiation, this value applies only for heavy particles (heavy ions, protons) and neutrons of all energies [7]. As can be seen in Fig.3, the $\Delta \mathrm{G}$ per unit fluence scales linearly with NIEL for heavy particles and neutrons; but, it follows a quadratic dependence on the NIEL for both electrons and gamma rays. To the contrary, when the $\Delta \mathrm{G}$ per unit fluence is plotted as a function of the effective NIEL for electrons and our ${ }^{60} \mathrm{Co}$ NIEL $\gamma$ value, evaluated using the effective NIEL curve $\left(\mathrm{T}_{\mathrm{d}, \mathrm{MD}}=1.617\right.$ $\mathrm{eV}$ ), the quadratic scaling becomes linear. This suggests that there is a reasonable agreement between experimental measurements and NIEL. However, Fig.3 shows a quadratic dependence of the $\Delta \mathrm{G}$ per unit fluence our ${ }^{60} \mathrm{Co}$ NIEL $\gamma$ calculation using the classical NIEL curves $\left(\mathrm{T}_{\mathrm{d}}=12.9 \mathrm{eV}\right.$ and $\mathrm{T}_{\mathrm{d}}=21$ $\mathrm{eV})$.

It is also noteworthy that the damage-factor values for ${ }^{60} \mathrm{Co}$ gamma-ray radiations in the work of Srour [7], were converted to an equivalent electron damage factor by using ${ }^{60} \mathrm{Co}$ NIEL $\gamma$ value taken from Summers et al [6], and this ${ }^{60} \mathrm{Co}$ NIEL $\gamma$ value is obtained by combining the slowed-down secondary electron spectrum with the electron NIEL based on BCA approach. In the present work, we used our ${ }^{60} \mathrm{Co}$ NIEL $\gamma$ value $\left(\mathrm{T}_{\mathrm{d}, \mathrm{MD}}=1.617 \mathrm{eV}\right)$ that provides a best fit to the experimental data. This ${ }^{60} \mathrm{Co}$ NIEL $\gamma$ value is obtained by combining the slowed-down secondary electron spectrum (Fig. 1) with the electron NIEL based on MD simulation [13]. In both works, it is considered that the displacement damage produced by ${ }^{60} \mathrm{Co}$ gamma-rays is due to energetic secondary electrons. The value of $K_{\text {dark }}$ calculated with this set of data derived from Ref. [7] and references cited therein, is $1.71 \times 10^{5}$ carriers $/ \mathrm{cm}^{3} . \mathrm{sec} /(\mathrm{MeV} / \mathrm{g})$, which is close to the one given by Srour [7] $\left(1.9 \times 10^{5}\right.$ carriers $\left./ \mathrm{cm}^{3} . \mathrm{sec} /(\mathrm{MeV} / \mathrm{g})\right)$.

\section{DISCUSSION}

Two main sources of error can limit the scope of the NIEL scaling approach:

i. The experimental conditions

ii. The threshold displacement energies used in the NIEL calculation.

Sufficient details of the test conditions for gamma irradiation unfortunately are often not mentioned in the literature. For example, is an equilibrium material placed in front of the test device or not during 
irradiation and, if so, what is the nature and the thickness of this equilibrium material? This is critical because the spectrum of secondary electrons that degrades the tested device strongly depends on the nature and the thickness of the materials which surround this device [18]. The lack of such detailed information introduces uncertainties into the determination of the ${ }^{60} \mathrm{Co}$ NIEL $\gamma$.

In the case of light particles such as electrons and gamma rays, the dependence of the NIEL on the threshold displacement energy is an important source of uncertainty. From Fig. 2 and Table 1, the NIEL is demonstrated to be strongly dependent on the value of $T_{d}$, and this parameter is poorly estimated for many materials. In fact, there is ambiguity about the meaning of $T_{d}$. The $T_{d}$ value is obtained by comparing the experimental defect introduction rate as a function of the incident particle energy with the calculated defect introduction rate for different $T_{d}$ values. But the data obtained have a large scatter. For instance, values of $T_{d}=12.9 \mathrm{eV}$ and $T_{d}=21 \mathrm{eV}$ are found for silicon [26, 27]. Both choices are considered because of the mathematical description used to fit experimental results.

The best agreement between the $\Delta \mathrm{G}$ per unit fluence that is evaluated at $300 \mathrm{~K}$ and 1 week after irradiation and predicted NIEL was obtained with our ${ }^{60} \mathrm{Co}$ NIEL $\gamma$ value calculated using the effective NIEL curve $\left(\mathrm{T}_{\mathrm{d}, \mathrm{MD}}=1.617 \mathrm{eV}\right)$. Therefore, if the damage factor is known for silicon device irradiated with a particular incident particle, the linearity obtained in Fig. 3 makes it possible to estimate the dark-current damage factor of other particles in different energies. However, this result can be controversial, because it isn't clear whether the universal value of $\mathrm{K}_{\text {dark }}$ that applies for heavy particles and neutrons, will also apply for low-energy electrons and ${ }^{60} \mathrm{Co}$ gamma rays.

The primary effect of displacement damage is the introduction of new energy levels in the Si bandgap. These defect levels can play an important role in changing the generation and recombination lifetimes. It was shown that those changes in electronic properties scale linearly with NIEL, especially for particles with relatively high NIEL values (i.e., $>2 \times 10^{-4} \mathrm{MeV} \mathrm{cm} \mathrm{/g)} \mathrm{that} \mathrm{primarily} \mathrm{produce}$ defect clusters in addition to isolated defects $[7,8]$. In that regime, $K_{\text {dark }}$ is shown to be independent of material and impurity type and concentration, and the universal value of $\mathrm{K}_{\text {dark }}$ applies for a various incident particles such as heavy ions, protons and neutrons [7]. For particles with relatively low NIEL (i.e., 1-MeV electrons and ${ }^{60} \mathrm{Co}$ gamma rays), only isolated defects are produced $[1,8]$. In that regime, the situation is more complex because there is likely to be a dependence of $\mathrm{K}_{\text {dark }}$ on material and impurity type and concentration [8]. Hence, a universal damage factor, $K_{\text {dark }}$, is not formally demonstrated to apply in this regime. More generally, a single value of $\mathbf{K}_{\text {dark }}$ that can describe both high and low-NIEL regimes needs confirmation. This hypothesis is largely based on the behavior of recombination-lifetime damage factor $\left(\mathrm{K}_{\mathrm{rec}}\right)$ for irradiated Si devices at relatively low NIEL, where the same behavior is assumed to occur for $\mathrm{K}_{\text {dark }}$ [8]. Systematic studies are required to confirm or deny this assumption. $\mathrm{K}_{\mathrm{rec}}$ displays several dependences in the low-NIEL regime, such as: material and impurity type, resistivity, oxygen concentration and injection level [8]. For high-NIEL regime, as discussed in 
[8], there are important similarities and differences between the behavior of $\mathrm{K}_{\mathrm{rec}}$ and $\mathrm{K}_{\mathrm{dark}}$. For instance, the dependence of $\mathrm{K}_{\mathrm{rec}}$ on dopant species, resistivity and injection level are absent in the case of $\mathrm{K}_{\text {dark }}$. Therefore, whether the behavior evident of $\mathrm{K}_{\mathrm{rec}}$ at low-NIEL regime will also exists in the case of $\mathrm{K}_{\mathrm{dark}}$ remains a very interesting open question.

To conclude, one cannot, at present, confirm or deny the potential dependence of $\mathrm{K}_{\text {dark }}$ on material and impurity type and concentration at low-NIEL values. Hence, our findings (Fig. 3) where the radiation-induced dark current in $\mathrm{Si}$ devices by means of increase in thermal generation in device depletion regions is described by a single value of $\mathrm{K}_{\text {dark }}$ for all kinds of particles (i.e., high and lowNIEL regimes), may be reasonable given the existing possibilities. However, a systematic study is needed to understand and clarify the behavior of $\mathrm{K}_{\text {dark }}$ at low NIEL regime.

There is another approach developed by the NRL group [14-16], which is an accepted method to eliminate the discrepancies observed between experimental data and predicted NIEL using a quality factor Q (E) in Eq. (1). This is defined as:

$$
Q(E)={\frac{\operatorname{NIEL}(E)}{\operatorname{NIEL}\left(E_{\text {ref }}\right)}}^{n 1}
$$

Where NIEL $\left(\mathrm{E}_{\mathrm{ref}}\right)$ is the NIEL value for a reference particle with a reference energy (e.g., $1 \mathrm{MeV}$ electrons), the " $n$ " factor is the fitting parameter used to make the experimental data agree with NIEL calculations. The value of $n$ is experimentally determined, but it depends on device type and parameter measured. Its value ranges between 0.5 and 3 [16], and will also depend on the experimental conditions that could alter the secondary electrons spectrum. This quality factor must be introduced into the calculation whenever the experimental degradation measurements do not scale linearly with NIEL. It is to be seen whether the method proposed in this present work holds for other damage parameters and is able to remove the need for a variable, $n$, in properly posed experiments.

\section{CONCLUSION}

The NIEL values for ${ }^{60} \mathrm{Co}$ gamma rays in silicon have been computed using the Monte Carlo radiation simulation code Geant 4 to estimate the slowed down spectrum of secondary electrons. Different electron NIEL curves from classical NIEL and "effective" NIEL have been also used for the calculation. Sources of error for the ${ }^{60} \mathrm{Co}$ NIEL $\gamma$ scaling approach were discussed, and it was demonstrated how the appropriate NIEL values can be used for predicting the ${ }^{60} \mathrm{Co}$ gamma rayinduced radiation damage in silicon devices without the use of a power factor $n$. It was found that the radiation-induced increase in thermal generation rate per unit fluence, evaluated at $300 \mathrm{~K}$ and 1 week after irradiation, scales linearly with our ${ }^{60} \mathrm{Co}$ NIEL $\gamma$ value calculated using the effective NIEL curve. However, more work needs to be done to fully investigate the behavior of $K_{\text {dark }}$ for irradiation with relatively low NIEL particles. 


\section{REFERENCES}

[1] J. R. Srour, C. J. Marshall, and P. W. Marshall, "Review of displacement damage effects in silicon devices,” IEEE Trans. Nucl. Sci., vol. 50, no. 3, pp. 653-669, Jun. 2003.

[2] J. R. Srour and J. M. McGarrity, "Radiation effects on microelectronics in space," Proc. IEEE, vol. 76, no. 11, pp. 1443-1469, November 1988.

[3] Jayashree, B.; Meulenberg, A.; Ramani; Radhakrishna, M.C.; Khan, S.A.; "Effects of nonpenetrating lithium-ion radiation on Si solar cells," Proc. 9th European Conference on RADECS, pp. 1-6, 2007.

[4] A. Meulenberg, F. C. Treble, "Damage in silicon solar cells from 2-155 MeV protons", Proc. 10th IEEE Photovoltaic Specialists Conf. (PVSC), vol. 13, no. 15, pp. 359, Nov .1973.

[5] G. P. Summers, C. J. Dale, E. A. Burke, E. A. Wolicki, P. W. Marshall, and M. A. Gehlhausen, "Correlation of particle-induced displacement damage in silicon," IEEE Trans. Nucl. Sci., vol. 34, pp. 1134-1139, Dec. 1987.

[6] G. P. Summers, E. A. Burke, P. Shapiro, S. R. Messenger, and R. Walters, "Damage correlation in semeconductors exposed to gamma, electron and proton radiations," IEEE Trans. Nucl. Sci., vol. 40, no. 6, pp. 1372-1379, Dec. 1993.

[7] J. R. Srour and D. H. Lo, "Universal damage factor for radiation-induced dark current in silicon devices,” IEEE Trans. Nucl. Sci., vol. 47, no. 6, pp. 2451-2459, Dec. 2000.

[8] J. R. Srour and J. W. Palko, "A framework for understanding displacement damage mechanisms in irradiated silicon devices,” IEEE Trans. Nucl. Sci., vol. 53, no. 6, pp. 3610-3620, Dec. 2006.

[9] P. Arnolda, C. Inguimbert, T. Nuns, and C. Boatella-Polo, "NIEL scaling: comparison with measured defect introduction rate in silicon,” IEEE Trans. Nucl. Sci., vol. 58, no. 3, pp. 756-763, Jun. 2011.

[10] A. Akkerman, J. Barak, M. B. Chadwick, J. Levinson, M. Murat, and Y. Lifshitz, "Updated NIEL calculations for estimating the damage induced by particles and gamma-rays in Si and GaAs," Rad. Phys. and Chem., vol. 62, pp. 301-310, 2001.

[11] C. Inguimbert, and R. Gigante, "NEMO: A code to compute NIEL of protons, neutrons, electrons, and heavy ions,” IEEE Trans. Nucl. Sci., vol. 53, no. 4, pp. 1967-1972, Aug. 2006.

[12] A. Meulenberg, "Proton damage to Silicon from Laboratory and Space Sources," Proc. The 12th IEEE Photo-voltaic Specialists Conf. (PVSC), Baton Rouge, LA, November 15-18, 1976,

[13] C. Inguimbert, P. Arnolda, T. Nuns, and G. Rolland, "Effective niel in silicon: Calculation using molecular dynamic results," IEEE Trans. Nucl. Sci., vol. 57, no. 4, pp. 1915-1923, Aug. 2010. 
[14]M. A. Xapsos, G. P. Summers, C. C. Blatchley, C. W. Colerico, E. A. Burke, S. R. Messenger and P. Shapiro, "CO60 Gamma Ray and Electron Displacement Damage Studies of Semiconductors," IEEE Trans. Nucl. Sci., vol. 41, no. 6, pp. 1945-1949, Dec. 1994.

[15]S. R. Messenger, M. A. Xapsos, G. P. Summers, and E. A. Burke, "Co60 gamma ray irradiations of solar cells: A new way to predict space radiation damage," in Proc. IEEE 1st World Conf. Photovoltaic Energy conversion, 1994, vol. 2, pp. 2153-2156.

[16] S. R. Messenger, G. P. Summers, E. A. Burke, R. J. Walters, and M. A. Xapsos, "Modeling solar cell degradation in space: A comparison of the NRL displacement damage dose and JPL equivamlent fluence approaches,” Progr. Photovolt.: Res. Appl., vol. 9, pp. 103-121, 2001.

[17] C. Inguimbert, and S. Messenger, "Equivalent displacement damage dose for on-orbit space application,” IEEE Trans. Nucl. Sci., vol. 59, no. 6, pp. 3117-3125, Dec. 2012.

[18] E. El Allam, C. Inguimbert, T. Nuns, A. Meulenberg, A. Jorio, and I. Zorkani, "Gamma and Electron NIEL Dependence of Irradiated GaAs ,” IEEE Trans. Nucl. Sci., vol. 64, no. 3, pp. 991-998, Mar. 2017.

[19] A. Meulenberg, "Ionization-Induced Damage in Crystalline Silicon", Proc. High Efficiency and Rad. Damage Silicon Solar Cell Workshop NASA/LeRC, pp. 221, 1977.

[20] A. Meulenberg, "Evidence for a Permanent Single-Event Upset Mechanism," IEEE Trans. Nucl. Sci., vol. 6, NS-31, pp. 1280-1283, Dec. 1984.

[21] [Online]. Available: http://geant4.web.cern.ch/geant4/

[22] ASTM E666-03, "Standard Practice for Calculating Absorbed Dose From Gamma or X Radiation", Annual Book of ASTM Standards, vol. 12.02.

[23] SR-NIEL: Screened Relativistic Nuclear Stopping Power Calculator. [Online]. http://srniel.mib.infn.it/index.php.

[24] I. Jun, W. Kim, and R. Evans, “Electron nonionizing energy loss for device applications,” IEEE Trans. Nucl. Sci., vol. 56, no. 6, pp. 3229-3235, Dec. 2009.

[25] A. Shatalov, S. Subramanian, and A. Klein, "Correlation between nonionizing energy loss and the offset voltage shift in InP-InGaAs heterojunction bipolar transistors,” IEEE Trans. Nucl. Sci., vol. 48, no. 6, pp. 2262-2269, Dec. 2001.

[26] J.W. Corbett and G.D. Watkins, "Production of Divacancies and Vacancies by Electron Irradiation of Silicon," Phys. Rev., vol. 138, no. 2A , pp. A555-A560, Apr. 1965.

[27] J. J. Loferski and P. Rappaport,"Displacement Thresholds in Semiconductors," Journal of Applied Physics, vol. 30, no. 8, pp. 1296-1299, 1959. 